\title{
Electrical Signs of Language in the Brain
}

\author{
Marta Kutas \\ Departments of Cognitive Science and Neurosciences \\ University of California, San Diego \\ Mireille Besson \\ Laboratoire des Neurosciences Cognitives \\ CN.R.S., Marseille
}

\section{Introduction}

How is knowledge represented in the brain? Does our knowledge of language differ in any substantive way from our knowledge of other domains such as music, for example? Are musical and linguistic chunks different? Do they interact according to a different set of principles or rules? What constitutes evidence of structural or functional identity in the case of music and language where complete identity is out of the question? Music and language are clearly different in many ways; nevertheless, they enjoy enough similarity to sustain research on the closeness of their representations and the computations in which they partake. We can ask similar questions within the domain of language for semantic versus syntactic representations, for example - for which the differences may be less obvious or less numerous than between language and music. We believe that adequate answers to such questions call for crossdisciplinary collaboration between anthropologists, cognitive scientists, cognitive neuroscientists, linguists, and psychologists. Morever, it requires the investigation not only of the structures and functions of many different languages, but those of the brain as well.

Our brains are on the one hand highly structured and on the other hand remarkably plastic. Their basic structure is imposed by the sensory input receptors (e.g., eyes, ears, nose, skin) and the motor effectors (e.g. hands, feet, tongue), but the fine structure arises out of a lifetime of experiences - spatial and temporal co-occurrences of neural activity of various orders and on multiple time scales. Which experiences are relevant to language 
comprehension? As we use language to convey meaning, which is admittedly an elaborate construction in our minds from the cues in the linguistic stream, much of what we experience (conscious or unconscious) must be relevant to how we understand and use language. It is also probable that learning one language rather than another has consequences on processing. Languages vary in what information is readily available in the linguistic input (e.g. English has almost no morphological markers for case), in the locus and nature of their linguistic ambiguities, and consequently in the demands that various of their linguistic analyses make upon working memory processes. Insofar as this is the case, the specifics of any given language must have an impact on how quickly comprehension unfolds, the types of information that are actually used in on-line processing and their relative contribution to the various and sundry linguistic operations as well as the types of misunderstandings that a reader or listener is likely to experience.

Language processing is constrained in certain ways in part because meaning is constructed via analyses in a relatively short-lived, capacity-limited working memory system; this system uses associatively- and categorically-based, hierarchically-organized sensory, conceptual, and motor memory systems, and is implemented in a brain with many hundreds of areas that perform different functions and are subject to the organizational principles of the neocortex (if we can use the 30 or so areas used by the visual system as an estimate). Thus the structure of the input and that of the processing medium mutually constrain each other in order to determine what information is processed and what information is lost. This interaction also determines which signals follow which paths from the sensory receptors to higher brain areas, whether or not signals are fed back from higher to lower areas to sustain or highlight the initial activation, or to inhibit local neighbor cells with different response properties, etc., and thereby determine what we perceive, think, and ultimately remember.

\section{The brain's electrical activity}

One way to look at the relations among the input, the physiology of the brain, and what we understand is to record the patterns of electrical activity at the human scalp. As long as the brain is functioning, there is some ongoing electrical activity, for the brain's currency is electrochemical. Unlike positron emission tomography (PET) and functional magnetic resonance imaging (fMRI), both the electroencephalogram (EEG) and its magnetic counterpart, the magnetoencephalogram (MEG) are direct measures of the electrochemical activity by which brain cells (neurons and glia) communicate. The neuroimaging measures 
mentioned previously as well as others based on metabolism or hemodynamic changes are at best of secondary importance for neuronal activity.

An event in the world - be it a light, a sound, a touch, a smell, or a cognitive event such as a decision or an intention to move - is reflected in the concerted activity of neurons in various parts of the brain. While the brain's response to any one specific input may be difficult to discern among all else that is going on, repeating the event (or categories of like events) and averaging across these repetitions allows the signal to emerge from the noise. The signal is a waveform of the voltage across time from the moment the stimulus is presented, although a few hundred milliseconds of prestimulus activity is recorded to serve as a baseline for analytic purposes. The waveform consists of peaks and troughs, negativities and positivities relative to the baseline (see Figure 1), although polarities are not absolute, but rather relative to the location of the two electrodes that serve as inputs to the differential amplifier that allows us to see these $1-60 \mathrm{uV}$ fluctuations in the EEG.

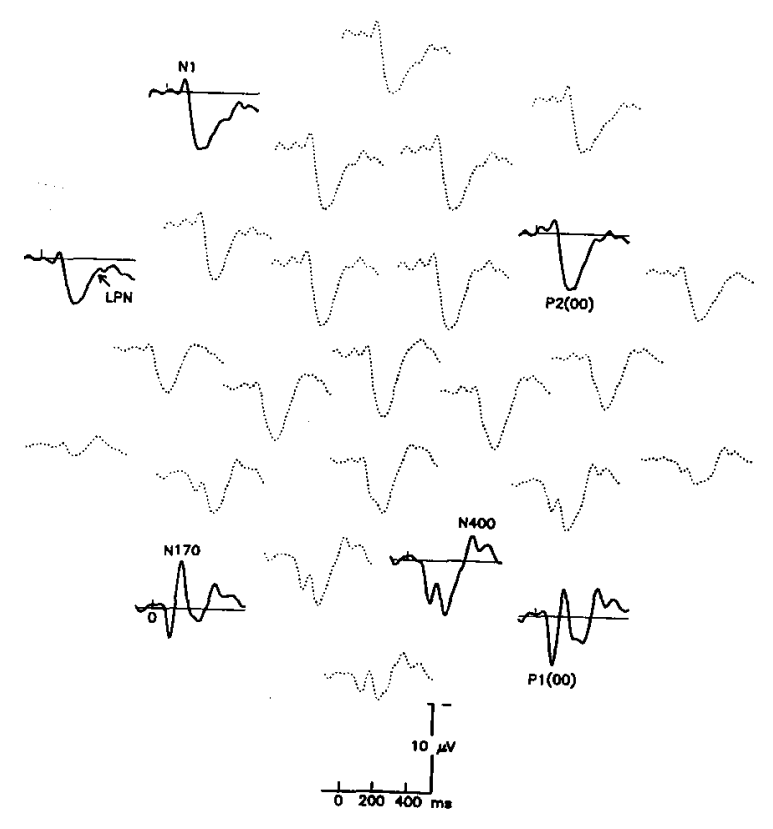

Figure 1. Grand average (across stimuli and subjects) ERPs elicited by the first noun in a set of sentences at 26 different recording locations across the scalp. Top 
represents the front of the head, bottom the back of the head. Some of the typical components (peaks or troughs) elicited by various visual stimuli (e.g. N1, PI (OO), N170, P2(00), lexical processing negativity or LPN, and N4OO) are labelled at characteristic sites. Zero represents stimulus onset. All waveforms on this and all subsequent figures are plotted with negative upwards.

Not all brain activity is volume-conducted to the surface in order to be detectable by an electrode at the scalp. For example, the activity of closed-field configurations of neurons are not; only open-field configurations of activity are volume-conducted to the surface. The neocortex, however, is essentially an open field in its functioning and meets all the necessary criteria for producing an externally observable electric potential: (1) the average distribution of sources and sinks in a patch of cortex is distributed in a non-radially symmetrical fashion, (2) the neurons are aligned in some systematic fashion, and (3) the neurons are activated in a synchronized fashion. About $70 \%$ of the cells in the neocortex are pyramidal cells; these have apical dendrites that extend from the soma towards the surface and give the cortex a columnar appearance. When these apical dendrites or the cell body are activated, the current flow in and out of the cell creates an approximately dipolar source/sink configuration, oriented perpendicularly to the cortical sheet, with its orientation determined by the actual site of the stimulation. The potential field produced by any single

cortical pyramidal neuron is quite weak; however, those produced by a patch of cortex containing hundreds of thousands of such pyramidal cells can sum and produce a strong field that can be detected at the scalp. This is, in fact, primarily what we see reflected in the scalp-recorded ERPs. In short, most of the observed electric recordings at the scalp are generated by post-synaptic currents in the apical dendrites of cortical pyramidal cells in the neocortex. Being limited to seeing the activity in the neocortex is not really so limiting after all: the neocortex is the principal neural substrate of perceptual processing, is involved crucially in motor execution and planning, and underlies much of what we mean by 'higher cognition' including language.

\section{What can we learn from ERPs?}

Let us first examine how one reads ERPs and makes inferences from one or more. Generally, one starts with a waveform of voltage in time where zero represents stimulus onset. It is now commonplace to look at multiple (e.g. 12128) waveforms of this kind recorded concurrently from different scalp locations to get an idea of how the waveform changes not only in time but in space (see Figure 1). Insofar as the distribution of an ERP waveform reliably 
changes across time, one must infer that more than one generator was active. Knowing exactly how many generators were active at any given moment would require special spatial-filtering algorithms, but the fact that there was more than one appears in changes in distribution. Classically, peaks are labelled and identified as components, which can be shown to be reliably correlated with particular experimental manipulations; although, in fact, there is nothing special about peaks as opposed to other parts of the waveform. The word component is variably used by different researchers to refer to (1) a peak, (2) a waveform feature whose polarity, latency, and scalp distribution are predictable from experimental manipulations, or (3) the reflection of the activity of a particular generator or set of generators in the brain. Since a peak is often correlated with a particular cognitive process, it has been assumed that it can be used as a physiological index of that process with its timing inferred from the latency of the peak (propagation of the potential field is essentially instantaneous), and with its degree of activation or 'strength' (somewhat mistakenly) inferred from the amplitude or area under the peak.

If two ERP waveforms differ significantly, then it is generally safe to assume that the brain activity and associated mental activities are different in the two conditions. The exact nature of the difference at the scalp is very much a function of where the recording electrodes are situated relative to the underlying generators. Also, it is important to note that one cannot assume that the electrode site where a potential is the greatest necessarily provides unequivocal information about the location of the underlying generator. One can also make reasonable inferences from the time course of the differences between the ERPs from two conditions, using the point at which two waveforms first differ as an upper limit on the time by which the brain has detected the difference between the eliciting stimuli.

\subsection{Is language special?}

Now that we know how to look for changes in the ERP, let us see what effects have been observed and what they might tell us about how special language really is: that is, what they have to say about the general question of whether language is separate from knowledge about other domains such as music (which we will discuss later) and the more limited question of whether different sorts of linguistic knowledge are represented differently and independently in the mind/brain.

The most relevant transient potentials are the N400, the N280 (more generally known as the lexical processing negativity - LPN) and the left anterior 
negativity (LAN), and the P600 or syntactic positive shift (SPS). The N400 is a negative potential between 100 and $600 \mathrm{~ms}$ with a peak around $400 \mathrm{~ms}$ after the onset of a word or pseudo-word, which provides a measure of how and when semantic constraints are imposed by context on the processing of an item. The LPN is a negativity whose latency varies between 250 and $350 \mathrm{~ms}$ as a function of the frequency of occurrence of the eliciting word in the language. The LAN is a negativity between 200 and $700 \mathrm{~ms}$ after a word onset which has been hypothesized to reflect working memory load. The P600 is a positivity between 200 and $800 \mathrm{~ms}$ which varies with manipulations of various syntactic constraints and their violations, and is correlated with processing difficulty due to structural factors. These are considered to be different components or effects, because they differ in their scalp distributions and because they differ in their sensitivities to experimental manipulations. The N400 shows the largest amplitude over posterior sites of the right hemisphere. The LPN has a maximal amplitude over left-anterior sites. The LAN is maximal over the frontal-central regions of the left hemisphere, and especially large over prefrontal areas. The P600 is very broadly distributed, often but not always maximal over bilateral posterior areas.

\subsection{Different representational levels in language}

So how can these potentials or effects be used to distinguish between representational levels in language? There have been three primary approaches. One is to violate various representational levels and see if the brain's response, in this case the ERPs, is similar or different; differences implicate the involvement of different brain areas and the existence of different levels. Here the search has been for representation-specific ERP components. In the same way, researchers have sought language-specific ERP components to support hypotheses about the modular nature of the language processor. Later we will examine this in a direct comparison of ERPs to structural violations in music versus those in language. A second approach has been to contrast the brain's responses to different categories of words such as open- and closed-class words and to compare their relative distribution, as distinct scalp topographies are usually taken to strongly suggest different neural generators. A third main approach has been to look for interactions between levels of representation; that is, to look for evidence of context effects in purportedly modular processes. This has often involved determining whether and under which circumstances higher level contextual information influences processing at lower levels. 


\subsection{Semantics and syntax}

\subsubsection{The N400 component}

Much effort has gone into identifying the distinct components in the ERP which index syntactic versus semantic processing. Lexical semantic violations as in I take coffee with cream and dog as opposed to sugar elicit an N400 (Kutas and Hillyard 1980). N400s are largest for outright violations whether in written or spoken English, Spanish, French, German, Dutch, or Japanese and even American Sign Language (for review see Kutas and Van Petten 1994). Its amplitude is inversely related to the cloze probability of a word in a sentence context (Kutas and Hillyard 1984; Kutas, Lindamood and Hillyard 1984).

In fact, the response to each word has an N400 whose amplitude is determined by its semantic expectancy, which is set up by the sentence context. This was shown by Van Petten and Kutas $(1990,1991)$ who compared the effect of context buildup on the N400 for words in normal congruent sentences, those with only syntactic structure but no meaning, and completely scrambled sentences without meaning or structure. Only the congruent sentences showed a modulation of the amplitude of the N400, becoming progressively smaller over the sentence as the semantic context built up. They also showed a similar interaction between word frequency and semantic context on N400 amplitude, but again only for meaningful sentences; the effect of frequency is greater at the beginning of a sentence, but diminishes as semantic constraints build up toward the end of the sentence (Van Petten and Kutas 1990, 1991).

\subsubsection{The P600 Component}

Let us examine the types of ERP responses that have been reported for other types of violations (other than semantic) within sentences. The first direct comparison of lexico-semantic and morphosyntactic violations indicated that various morphosyntactic violations in English elicited a late positivity (a result subsequently replicated in Spanish), which has since been called a P600 or SPS, as well as some anterior negativities - but not any substantive N400 activity (Kutas and Hillyard, 1983). In subsequent reports, this positivity has been interpreted as indexing (directly or indirectly) the operation of a modular syntactic parser. Various other types of syntactic violations have since been used in English, Dutch, Finnish and German. For example, in addition to violations in subject/verb agreement, researchers have investigated violations of subcategorization constraints, violations of phrase structure, and violations of case markings. For example, Münte and Heinze (1994) found a P600 to the 
inappropriate genitive case marking on the word Besens in Die Hexe benutzte ihren Besens, um zum Wald zu fliegen ("The witch used her broom's to fly to the forest") relative to the correct word Besen (see Figure 2).

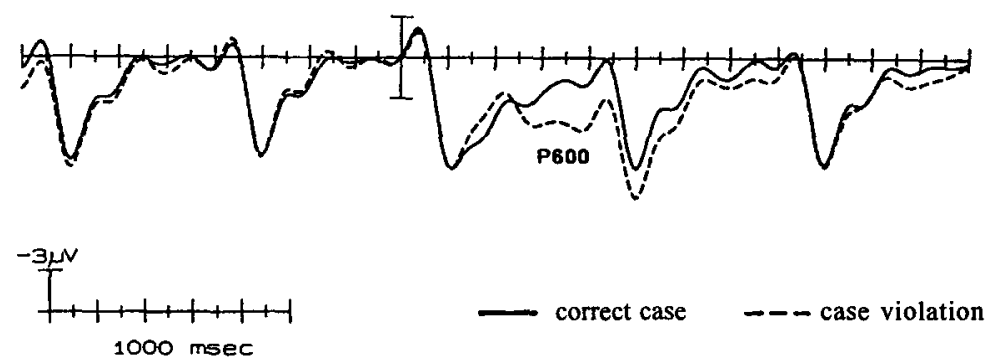

Figure 2. Comparison of the grand average ERPS to a five-word segment of German sentences containing syntactic violations (dashed line) with those containing the syntactically correct forms (solid line); these sentences were read one word at a time. The recording was taken from an electrode at the top of the head (vertex). Figure adapted from Münte and Heinze (1994).

The presence of N400s in response to lexical semantic violations and P600s in response to many (although not all) types of syntactic violations has been offered as evidence for a corresponding division between semantic and syntactic processors (e.g. Osterhout and Holcomb 1992). But subcategorization violations in Dutch do not elicit a P600. Should this result be considered an indicator of a linguistic difference? Moreover, the various positivities (so-called P600s) in the different experiments varied in their scalp distributions and their relative latencies. These differences in scalp distribution imply different configurations of neural generators. Thus, the P600 does not appear to be a unique index of syntactic processing. Moreover, Coulson, King and Kutas (1998) showed that the amplitude of the P600 (like that of the P300, elicited by task-relevant, unexpected non-language events) is sensitive to the probability of violations. That is, if syntactic violations are frequent and the syntactically correct form is infrequent, then the difference in the P600s between the two conditions is much less than if the syntactic violation also occurred infrequently. The non-additivity of the effects of syntactic violations and frequency of occurrence of the violation argues against the independence of their generators. 


\subsubsection{The Left Anterior Negativity and N280}

In fact, the left-anterior negativity (LAN) as in WH-movement seems to be a more consistent response to syntactic violations. At present however, it is unclear how it differs from a similar negativity seen in a number of situations where there are no syntactic violations, but merely loads on short-term memory such as in WH-questions in English. Kluender and Kutas (1993a) hypothesized that the LAN reflected cognitive operations involved in entering a filter into working memory, storing it, and subsequently retrieving it to assign fillers to gaps (also see King and Kutas 1995a).

In a second approach, cognitive electrophysiologists have also contrasted scalp distributions of various lexical classes such as open-class versus closedclass words. The idea is based on the loose association of open-class words with semantics and closed-class words with syntax. This division has been supported electrophysiologically by the argument that the response to openclass words has a large N400 component over right-posterior sites and that the response to closed-class words has a large negativity (N280) over left-frontal sites (near Broca's area). However, even this division is questionable. First, N400s are not restricted to open-class words. For instance, Kluender and Kutas (1993b) observed N400s in response to the closed-class words that, if, and who in questions like Can't you remember that/if/who, with the largest N400 to who. Moreover, there are data indicating that not all open-class words show a large N400 component. For example, a semantic violation repeated for the second time does not elicit an N400 response (Besson et al. 1992), nor does a content word at the end of a sentence (Van Petten, 1993; Van Petten and Kutas 1990; 1991). Closed-class words are more predictable from context than openclass words, and we believe this difference in contextual predictability explains why open-class words generally have larger N400 components.

It is also possible to show that N280 responses are not specific to closedclass words. In fact, the ERPs to all words over left-frontal regions contain a negativity; however, its latency varies with the eliciting word's frequency. Figure 3 illustrates the lexical processing negativity (LPN) to definite articles, adverbial prepositions, adjectives, verbs and nouns. 


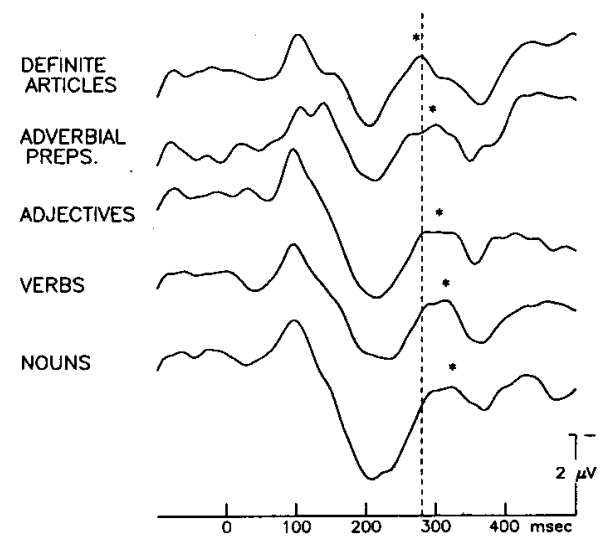

Figure 3. Grand average ERPs (across 24 subjects) recorded over a frontal site in the left hemisphere (F7) for representative word types that are subclasses of the broad Open Class (adjectives, verbs, nouns) versus Closed Class (definite articles, adverbial prepositions). The dashed line is at $280 \mathrm{~ms}$ post-word onset; asterisks mark peak latencies for the word types.

A linear regression of the peak latency of the LPN and word scarcity (a transformation of frequency) accounts for, on average, $90 \%$ of the variation. Thus, the LPN is definitely not a marker for closed-class words; it is present with the same distribution across the scalp for both word classes, but at a shorter latency for the higher frequency closed class words than for the lower frequency open-class words. And, a $50 \mathrm{~ms}$ processing difference of this sort may well lead to real functional differences in the processing of open- versus closed-class words, which obviates the need for the proposed special closedclass processor (located in Broca's area) to account for the observed differences (King and Kutas 1995b, 1998).

While neither the N400 nor the LPN are specific to any lexical class, they can, nevertheless, be used to investigate linguistic representations. We have already mentioned that semantic context modulates N400 amplitude in sentences. N400s are also sensitive to category membership (e.g. Fischler et al. 1983; Boddy and Weinberg 1981; Heinze et al. 1998). Following the presentation of a word belonging to a semantic category, responses to members of the category have much smaller N400s than do non-members. Moreover, the ERPs of more typical members have smaller N400s than those to less typical members. Kara Federmeier took advantage of the fact that both sentential context and category membership influence N400 amplitude, to investigate how 
constraints arising from the organization of categories in long term memory interact with constraints arising from sentence fragments during on-line reading. She asked people to read sentences that ended either with the name of an object predicted by the context, the name of an object from the same category but not predicted by the context, or an unexpected name of an object from a different category. For example, Checkmate! Rosaline announced with glee. She was getting to be really good at CHESS (expected), MONOPOLY (same category), and FOOTBALL (different category). The preliminary results showed that the ERP corresponding to the expected ending included a large positivity between 300 and $600 \mathrm{~ms}$, whereas both unexpected endings were characterized by N400 responses - the ERP to the item from the different category being larger. It seems that expectations as people read are not only for specific words but also for more general categories. These results suggest that items from the expected taxonomic category are more easily integrated even if they are not more plausible, which is consistent with the hypothesis that context operates through the use of category structure for objects in long-term memory. We are now looking for similar effects using pictures of objects and categories of actions.

\subsubsection{The Slow Potentials}

By focusing on the response to various types of violations, the emphasis in ERP studies of language has remained at the level of single words, even if these words occur within a sentential context. This is like the majority of neuroimaging investigations of language, which have been based mostly on isolated words taken from word lists. But language is more than this, involving not only the registration of single words but also the computation of the syntactic, semantic, and thematic relationships among words. Moreover, at least some of these processes must extend temporally over a series of processing events such as for anaphoric reference and the comprehension of WH-questions in English. ERPs are well-suited to track these extended brain processes.

Such slow processes are reflected in slow potentials and revealed by applying a low-pass digital filter to the cross-sentence ERPs, so that only the slow activity remains. Even after filtering, there remains a temporally and spatially rich and complex pattern of electrical activity over the head; these are potentials that fluctuate with the structure of the clause and the functional structure of the brain. As simple as these sentences are, they still require analysis at multiple linguistic levels and must utilize working and long-term memory to be understood. 
There are many features to these slow potentials that allow one to track the course of the processing of a clause. In brief, we have identified four laterally asymmetric slow potential effects (see Figure 4):

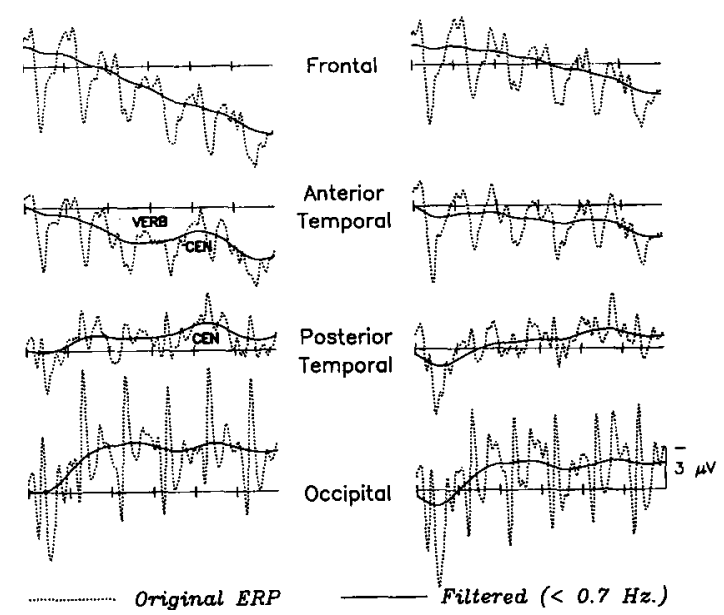

Figure 4. Original ERPs (dotted line) recorded from 18 subjects, and slow potential activity (solid line), low pass-filtered at $0.7 \mathrm{~Hz}$ for 4 pairs of sites going from front to back of the head over left and right hemispheres, shown in the left and right columns, respectively. The ERP reflects the response to the first six words of a transitive clause (e.g., The secretary answered the phone because...). The region of the verb and the clause ending negativity or CEN are labelled. Reprinted by permission of MIT Press, Kutas and King (1996).

(1) A sustained negative shift over occipital sites associated with early visual processing,

(2) a temporal positivity reflecting verb processing (perhaps thematic role assignment),

(3) a negativity over temporal sites indexing clause ending (clause ending negativity or CEN), and

(4) a very slow-going frontal positivity that might reflect the building of a mental model or schema of the sentence from an integration of items in working memory with representations in long-term memory.

Moreover, all of these are taking place simultaneously but over different brain regions with different time courses.

We have recorded similar slow potential effects for a variety of sentence structures such as subject- and object-relative constructions (e.g. subject 
relatives: The reporter who harshly attacked the senator admitted the error. versus object relatives: The reporter who the senator harshly attacked admitted the error.). Without going into great details, King and Kutas (1995a) found that the processing of these two differ and these differences can be linked to differences in their demands on working memory. Moreover, we have found that the effects were very similar, whether the sentences were written or spoken (Müller, King and Kutas, 1997). Perhaps most important for present purposes is the fact that these can be used to examine potential interactions between supposedly different linguistic levels of analysis. For example, Jill Weckerly (1995) used these to examine whether noun animacy (a semantic factor in English, although not in all languages) is used on-line during reading of sentences with object-relative constructions which varied only in the order of the animacy of the nouns in the main and relative clauses.

In brief, we observed many of the same cross-sentence patterns as in previous studies: namely, the ultra-slow frontal positivity, the verb positivity, the CEN, and LAN effects on the verbs. Moreover, the brain was shown to be sensitive to noun animacy as soon as the noun was read, which had consequences on the upcoming ERPs including the complementizer, the relative clause subject, and the relative and main clause verbs (see Weckerly and Kutas, in press). We have argued elsewhere that these data support our hypothesis that the animacy information is combined (1) with word order to make tentative grammatical role assignments and (2) with the complementizer (that) to set up expectations for the object relative construction. We thus have evidence that semantic and pragmatic knowledge are brought into the initial parsing analysis.

More generally, our results show cross-talk between supposedly separate, independent levels of linguistic analysis.

To sum up, we have shown how it is possible to examine the brain's activity during language processing via ERP recordings from the human scalp. Both word- and sentence-level responses reflect the brain's sensitivities to linguistic analyses of various types. To date, none of our data support any unequivocal presence of encapsulated linguistic representations that do not interact prior to their full processing. We have found no unique markers of any language-specific processes. Next, we will examine how the modularity hypothesis fares when we compare language versus music representations and processes.

\subsection{ERPs and music}

The first experiment that we conducted in music perception research (Besson and Macar 1987) was aimed at testing the functional significance of the N400 
component. We wanted to determine whether or not the presentation of an incongruous note (out of key) at the end of a musical phrase would elicit an $\mathrm{N} 400$, as does the presentation of an incongruous word at the end of a sentence. Our results show that only incongruous words are associated with N400s. Incongruous notes, in contrast, elicit late positive components (LPCs), peaking around $500 \mathrm{~ms}$ after terminal note onset over parietal sites. Should we then have concluded that N400 is language-specific? Several reasons led us to reserve judgment. First, musical phrases were highly familiar and incongruous notes were so obvious that they did not require any specific musical knowledge to be noticed. Furthermore, since participants were not musicians, they could not access musical knowledge. It is still an open question whether the N400 reflects the access to and the computations performed on knowledge, regardless of its content.

\subsubsection{Harmonic violations}

We conducted a second series of experiments (Besson, Faita and Requin, 1994; Besson and Faita, 1995) in which we manipulated:

- musical expertise: musicians with a classical background, professionals or with at least 7 years of training at the regional musical school and nonmusicians participated in the experiments;

- the familiarity of the materials: we used well-known musical phrases, from the classical repertoire (Vivaldi Four Seasons, Mozart Turkisch March, etc ... ), and new, unknown musical phrases, composed for the experiment following compositional rules of classical music;

- the type of incongruity: musical phrases, known or new, ended with a congruous note, an incongruous note out of key (nondiatonic) or in key but not as expected - as a function of the melodic contour (diatonic incongruity), or with a congruous note delayed by $600 \mathrm{~ms}$ (rhythmic incongruity). Note that each type of terminal note had the same probability of occurrence.

As illustrated in Figure 5, compared with congruous notes, nondiatonic incongruities elicited large LPCs, as was shown by Besson and Macar (1987). Diatonic incongruities also elicited LPCs, but of smaller amplitude than the nondiatonic incongruities. These effects were larger in size for known than for unknown melodies, and larger for musicians than for non-musicians. 


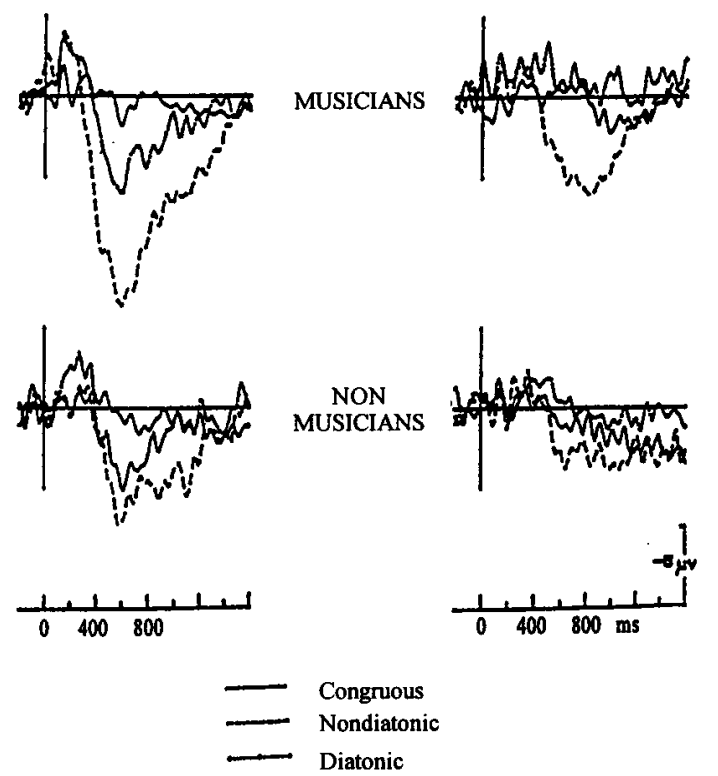

Figure 5: ERPs recorded from musicians (15 participants) and non-musicians (15 participants) in response to congruous note (solid line), nondiatonic incongruity (dashed line) and diatonic incongruity (dashed-dot line) ending known (left column) and unknown (right column) melodies. Recordings are from parietal site (Pz). Adapted from Besson and Faita (1995).

Therefore, nondiatonic and diatonic incongruous notes are associated with LPCs but not with the N400 component. As mentioned above, late positivities, such as P600, have also been elicited in response to syntactic incongruities. Thus, it may be that the similarities between language and music emerge at the level of harmonic and syntactic structures rather than at the level of semantic representations. Recently, this hypothesis was directly tested by Patel et al. (1998). Musicians were presented with linguistic and musical phrases, in which syntactic violations and harmonic violations were introduced, respectively. The results showed that the LPCs elicited in the two linguistic and musical conditions were not significantly different. 


\subsubsection{Rhythmic violations}

Results for rhythmic incongruity are presented in Figure 6. A large positive component is associated with the absence of the note; that is, this component appears when the terminal note should have been presented, but was not. The amplitude of this positive component does not differ for musicians and nonmusicians, but is larger for known than for unknown melodies.

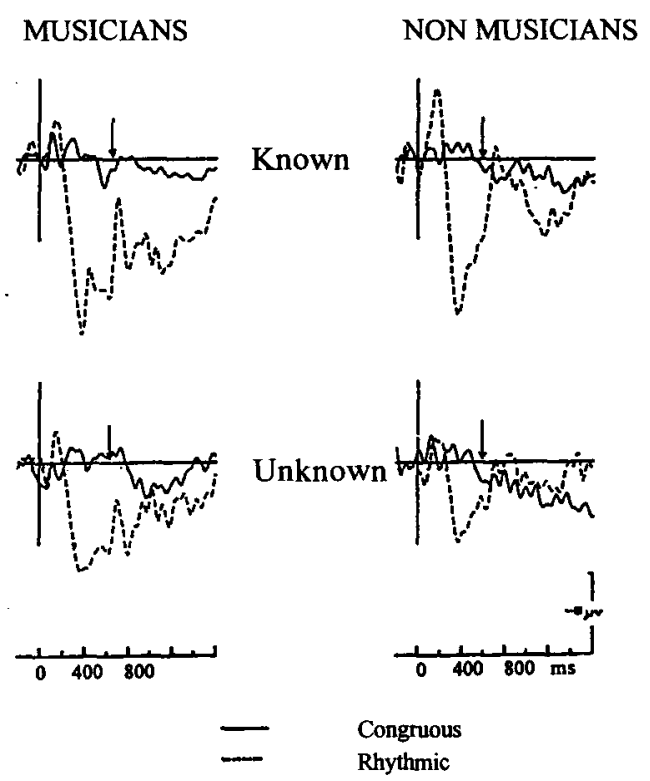

Figure 6: ERPs recorded from musicians (15 participants) and non-musicians (15 participants) in response to congruous note (solid line), and rhythmic incongruities (dashed line) ending known and unknown melodies. The vertical bar shows when the terminal note should have been presented, and the arrow when it was actually presented. Recordings are from parietal site (Pa). Adapted from Besson and Faita (1995).

These results demonstrate that ERPs are sensitive to rhythm and consequently, to the temporal structure of musical phrases. We recently conducted a series of experiments in which we added some unexpected pauses in written or spoken sentences, such that the sentence terminal word was either presented when it should have been, or with a $600 \mathrm{~ms}$ delay (Besson et al, 1997). The effect of temporal structure violation in spoken sentences was found to be very similar to that observed in previous music experiments. 
Therefore, it seems that temporal information is processed similarly in linguistic and musical phrases. Experiments are now in progress, using different brain imaging techniques, such as fMRI and MEG, to determine whether or not the same brain areas are activated by the processing of temporal information in both language and music.

\subsubsection{Lyrics}

In another series of experiments, we directly compared the effects of semantic and musical incongruities. To this end, we selected excerpts from French opera, which were then sung a capella by a professional singer. These excerpts lasted for 15-20 seconds, and ended either with a congruent word sung in or out of key, or with an incongruous word sung in or out of key (Bizet, Carmen: "The fabric floated in the breeze / blood"). They were presented to professional musicians from the Opera in Marseille who were asked to detect the linguistic and musical incongruities. Results showed that N400s were elicited by incongruous words, and that LPCs were elicited by words sung out of key. Most importantly, both components, the N400 and the LPC, were elicited by the double incongruity (incongruous words sung out of key). Thus, these results demonstrate the strong additivity of the ERP components associated with semantic and musical incongruities. As such they point to the independence of semantic and musical processing.

\section{Conclusion}

In conclusion, comparing language and music processing reveals both similarities and differences. Differences are mainly demonstrated when studying semantic representations and the computations that allow access to a word's meaning. On the other hand, similarities arise when the focus is on syntactic, harmonic, and temporal information processing. Overall, these results favor the idea according to which the cognitive operations that subserve language and music processing obey similar, if not common, functional principles. Our data are, therefore, inconsistent with a strong modular view of linguistic and musical processing. 


\section{Acknowledgments}

Some of the work reported herein was supported from grants NIMH (MH52893), NIA (AG08313), and NICHD (HD22614) to M. Kutas, and from grants MRT (92.C.0420) and G.I.S., "Science de la Cognition" (1995-1997) to M. Besson.

\section{References}

Besson, Mireille and Macar, Françoise. 1987. "An event-related potentials analysis of incongruity in music and other non-linguistic contexts". Psychophysiology, 24: 14-25.

Besson, Mireille, Kutas, Marta and Van Petten, Cyma. 1992. "An Event-Related Potential (ERP) analysis of semantic congruity and repetition effects in sentences". Journal of Cognitive Neurosciences, 4: 132-149.

Besson, Mireille, Faïta, Frederique and Requin, Jean. 1994. "Brain waves associated with musical incongruity differ for musicians and non-musicians". Neuroscience Letters, 168: 101-105.

Besson, Mireille and Faïta, Frederique. 1995. "An Event-Related Potential (ERP) study of musical expectancy: Comparison of musicians with non-musicians". Journal of Experimental Psychology: Human Perception and Performance, 21 (6): 1278-1296.

Besson, Mireille; Faïta, Frederique; Czternasty, Claire and Kutas, Marta. 1997. "What's in a pause: Event-Related Potential analysis of temporal disruptions in written and spoken sentences". Biological Psychology, 46, 3-23.

Boddy, John and Weinberg, Hal. 1981. "Brain potentials, perceptual mechanisms and semantic categorization". Biological Psychology, 12: 43-61.

Coulson, Seana; King, Jonathan W. and Kutas, Marta. 1998. "Expect the unexpected: Event-related brain responses to morphosyntactic violations". Language and Cognitive Processes, 13: 21-58.

Fischler, Ira; Bloom, Paul A.; Childers, Doug G.; Roucos, S.E., and Perry, Nathan W. 1983. "Brain potentials related to stages of sentence verification". Psychophysiology, 20: 400-409.

Heinze, Hans; Münte, Thomas and Kutas, Marta. 1998. "Context effects in a category verification task as accessed by Event-Related brain Potentials measures". Biological Psychology, 47: 121-137.

King, Jonathan and Kutas, Marta. 1995a. "Who did what and when? Using wordand clausal-level ERPs to monitor working memory usage in reading", Journal of Cognitive Neuroscience, 7(3): 376-395.

King, Jonathan and Kutas, Marta. 1995b. "A Brain Potential whose latency indexes the length and frequency of words", CRL Newsletter October, 10(1).

King, Jonathan and Kutas, Marta. 1998. "Neural plasticity in the dynamics of visual word recognition". Neuroscience Letters, 244: 61-64.

Kluender, Robert E. 1991. Cognitive Constraints on Variables in Syntax. Dissertation. University of California, San Diego. 
Kluender, Robert and Kutas, Marta. 1993a. "Bridging the Gap: Evidence from ERPs on the processing of unbounded dependencies". Journal of Cognitive Neuroscience, 5(2): 196-214.

Kluender, Robert and Kutas, Marta. 1993b. "Subjacency as a processing phenomenon". Language and Cognitive Processes, 8(4): 573-633.

Kutas, Marta. and Hillyard, Steven A. 1980. "Reading senseless sentences: Brain potentials reflect semantic incongruity". Science, 207: 203-205.

Kutas, Marta. and Hillyard, Steven A. 1983. "Event-Related brain potentials to grammatical errors and semantic anomalies". Memory and Cognition, 11: 539-550.

Kutas, Marta and Hillyard, Steven A. 1984. "Brain potentials during reading reflect word expectancy and semantic association". Nature, 307: 161-163.

Kutas, Marta; Lindamood, Timothy and Hillyard, Steven. 1984. "Word expectancy and event-related brain potentials during sentence processing". In S. Kornblum and J. Requin (eds), Preparatory states and processes (217 - 237). Hillsdale: Lawrence Erlbaum.

Kutas, Marta and Van Petten, Cyma. 1994. "Psycholinguistics Electrified: Eventrelated potential investigations". In M.A. Gernsbacher (ed.), Handbook of Psycholinguistics, Academic Press, 83-143.

Kutas, Marta and King, Jonathan W. 1996. "The potentials for basic sentence processing: differentiating integrative processes", In T. Inui and J.L. McClelland (eds.), Attention and Performance XVI. MIT Press.

Müller, Horst M., King, Jonathan W. and Kutas, Marta. 1997. "Event related potentials elicited by spoken relative clauses". Cognitive Brain Research, 5: 193-203.

Münte, Thomas and Heinze, Hans. 1994. "Event-Related negativities during syntactic processing of writtent words". In H. Heinze, T.F. Münte and G.R. Mangun (eds), Cognitive electrophysiology. Boston, MA: Birkhauser.

Patel, Ani; Gibson, Edward; Ratner, J.; Besson, Marta and Holcomb, Phillip. 1998. "Processing syntactic relations in language and music: An Event-Related Potential study". Journal of Cognitive Neurosciences, 10: 717-733.

Osterhout, Lee and Holcomb, Phillip. 1992. "Event-Related brain potentials elicited by syntactic anomaly". Journal of Memory and Language, 31: 785806.

Van Petten, Cyma and Kutas, Marta. 1990. "Interactions between sentence context and word frequency in event-related potentials". Memory and Cognition, 18: 380-393.

Van Petten, Cyma and Kutas, Marta. 1991. "Influences of semantic and syntactic context on open- and closed-class words". Memory and Cognition, 19: 95112.

Van Petten, Cyma. 1993. "A comparison of lexical and sentence-level context effects in event-related potentials". Language and Cognitive Processes, 8: 485-531. 
Weckerly, Jill. 1995. Object Relatives Viewed Through Behavioral, Electrophysiological, and Modeling Techniques. Dissertation. University of California, San Diego.

Weckerly, Jill and Kutas, Marta. "Electrophysiological analysis of animacy effects in the processing of object relative sentences". Psychophysiology, in press. 